http://jmscr.igmpublication.org/home/ ISSN (e)-2347-176x ISSN (p) 2455-0450 crossref DOI: https://dx.doi.org/10.18535/jmscr/v8i7.34

\title{
Role of Grey Scale and Color Doppler Ultrasound in Diagnosis of Lung Abscess
}

\author{
Authors \\ Maha M. ElKholy ${ }^{1}$, Hala A. Mohammad², Nasr M. Osman', \\ Bahaa Ibrahim. Mohamed ${ }^{2}$, Rasha M. Emam² \\ ${ }^{1}$ Department of Chest, Assiut University, Egypt \\ ${ }^{2}$ Department of Chest, Minia University, Egypt \\ ${ }^{3}$ Radiology Department, Minia University, Egypt
}

\section{Abstract}

The main purpose of this work is to compare the diagnostic accuracy (DA) of chest X ray (CXR), chest computerized tomography (CT)and lung grey scale ultrasonography (US)in evaluation of lung abscess and determine the role of color doppler ultrasound in diagnosis of lung abscess.

Sixteen patients with suspected lung abscess were evaluated by CXR, chest CT, grey scale and colour doppler chest sonography. Chest CT revealed the highest DA (98.5\%) with 100\% sensitivity, 98.3\% specificity, 88.9\% PPV and $100 \%$ NPV. The specificity, sensitivity, PPV, NPV and DA of chest $X$ ray were 98.3\%, 75\%, 85.7\%, 96.6\% and 95.5\% respectively. Grey scale US had the least sensitivity (53.3\%), specificity was 98.2\%, PPV 96.7\%, NPV $67.7 \%$ and DA 93.1\%. CDS showed absent vascularity were observed in 9 lesions (60\%), scanty and marked vascularity were equally detected each in 3 lesions (20\%) with 66.7\% showing THI flow and 33.3\% showing $\mathrm{MHI}$ flow. The present study revealed that chest CT is considered the most accurate radiological procedure for diagnosis of lung abscess. Although its low sensitivity, grey scale US has a relatively high specificity in diagnosing lung abscess.

Keywords: Lunggrey scale ultrasonography, Lung abscess, Chest X ray, Computed tomography, Color Doppler sonography.
\end{abstract}

\section{Introduction}

Lung abscess is defined as an area of suppurative or necrotic tissue in the lung parenchyma, which leads to a cavitation, and after development of bronchopulmonary fistula, an air-fluid level within this cavitary lesion (Seo et al., 2013). Acute lung abscess is usually circumscribed with not so well-defined surrounding to lung parenchyma, filled with thick necrotic detritus. Chronic abscess of the lung is usually irregular star-like shape with well-defined margin, filled with grayish thick necrotic tissues (Kuhajda et al., 2015).

The CXR is an old radiological procedure and also still the one most frequently used for diagnostic purposes in the pulmonary diseases (Wielpütz et al., 2014). Multidetector CT (MDCT) is widely distributed and is characterized by high, almost isotropic resolution (pixel size 0.5 to $1 \mathrm{~mm}$ in every spatial direction), allowing the image data to be seen in any plane desired (Biederer et al., 2008).

Ultrasonography can also be used to guide the sampling of lung abscesses to obtain biopsies for microbiological examination and to drain the pulmonary cavitary lesions (Patradoon-Ho and Fitzgerald, 2007). 


\section{Patients and Methods}

Sixteen adult patients from both outpatient chest clinic and inpatient chest department of Al-Minia university hospital from October 2013 to August 2016.This study was approved by ethics committee of the faculty of medicine of our university.

Study Inclusion Criteria: Patients aged $\geq 18$ years old with radiologically confirmed peripheral pulmonary lesions, and cooperative patients who are able to hold breaths temporarily to decrease interference from the chest wall movement while the flow signal was being acquired.

Exclusion Criteria: Patients with isolated pleural lesions, patients with chest wall lesions, possibility of ARDS, or patients with cardiac disease.

All studied patients were subjected to:

- Full history taking and clinical examination

- Laboratory tests including complete blood picture, sputum and pleural fluid examination and culture for acid fast bacilli and other pathogens.

- Chest radiographposterior-anterior view was done using a commercially available radiograph apparatus and a standard method (model Ralcos.r.i, model R302/A, Italy). The film was read by a good and an independent radiologist who was unaware of ultrasonoraphy and clinical data of our patients.

- Chest computed tomography (CT) scan was done. Either high-resolution CT, contrastenhanced CT scans or CT-pulmonary angiography were done according to the suspected diagnosis and analyzed by an independent radiologist, who was unaware of results of other tests and clinical data using a commercially available CT machine and a standard technique (model GE BRIGHT Speed 16 slices) (General electric Healthcare, USA). Reconstruction parameters were $5.0 \mathrm{~mm}$ slice thickness and medium smooth convolution kernel (B41s).

- Lung ultrasound was performed immediately after the chest radiograph with (a Philips, Clear Vue 350 Ultrasound Systems) using (2$5 \mathrm{MHz}$ ) convex probe for lung examination and $(5-12 \mathrm{MHz})$ linear probe for pleural evaluation. The examination of patients was done either supine or sitting position as clinically appropriate. First, Greyscale ultrasound was used to localize the entire lesion, and then a color Doppler sonography examination was added.

Sonographic findings were recorded including; lung sliding, pleural thickening, irregularities or effusion, presence of hypoechoic or isoechoic lesions or presence of air bronchogram.

\section{Methods of Statistical Analysis}

Data were collected, revised, verified, coded, then entered PC for statistical analysis done by using SPSS statistical package version 20.

For Quantitative Data: Mean (X ) and standard deviation (SD). For qualitative data: number (n) and percentage (\%). Kolmogorov- Smirnov for normality test was used to compare between parametric data and non-parametric data. Independent sample t-test for analysis of quantitative data. Chi square $\left(\mathrm{X}^{2}\right)$ test, Fisher Exact test for analysis of qualitative data. Cross tabulation was done for the estimation of sensitivity, specificity, PPV, NPV and DA.

\section{Results}

Sixteen adult patients were included in this current study; 10 males $(62.5 \%)$ and 6 females $(37.5 \%)$, their ages ranged from 18 to 56 years with a mean \pm standard deviation $(\mathrm{SD})$ of $45.6 \pm 11.54$ years. The demographic and clinical data of all studied patients were shown in table 1.

Cases were presented by cough and expectoration in $(93.3 \%)$, tachypnea in $(86.7 \%)$, toxic manifestations in $(73.3 \%)$, signs of consolidation in $(66.7 \%)$, fever in $(60 \%)$, dyspnea and chest pain each in $(40 \%)$, haemoptysis in $(33.3 \%)$, clubbing and signs of collapse each in (13.3\%) and signs of pleural effusion in $(6.7 \%)$. Low $\mathrm{SaO}_{2}$ was present in $(26.7 \%)$ of cases. 
The variable features of lung abscess by CXR, CT and grey scale US were demonstrated in table 2.

The most frequent CXR findings were air-fluid level $(75 \%)$, opacity with air bronchogram was present in $(37.5 \%)$, loss of lung volume and pleural effusion were equally presented each in $(6.25 \%)$ of cases.

Chest CT showed air-fluid level in all cases, then opacity with air bronchogram in $(75 \%)$ and associated pleural effusion was present in $6.25 \%$.

Grey scale US showed air bronchogram in $93.3 \%$, breath dependent motion in $86.7 \%$, pleural involvement in $26.7 \%$ and pleural effusion with internal echoes in $18.75 \%$, while one case $(6.75 \%)$ showed normal lung parenchyma.

Regarding colour doppler sonographic findings, $60 \%$ of cases showed absent vascularity, $20 \%$ had scanty vascularity and $20 \%$ had marked vascularity. Vascular flow signals showed that $66.7 \%$ of cases had THI flow and $33.3 \%$ had MHI.

Table (1): Demographic and clinical data of participant

\begin{tabular}{|c|c|c|}
\hline Variable & \multicolumn{2}{|c|}{$\mathrm{M} \pm \mathrm{SD}}$. \\
\hline Age (years) & \multicolumn{2}{|c|}{$\begin{array}{c}45.6 \pm 11.54 \\
(18-56 \text { years }) \\
\end{array}$} \\
\hline Variable & Frequency & $\begin{array}{c}\text { Percentage } \\
(\%)\end{array}$ \\
\hline $\begin{array}{c}\text { Gender: } \\
\text {-Males } \\
\text {-Females } \\
\end{array}$ & $\begin{array}{c}10 \\
6 \\
\end{array}$ & $\begin{array}{l}62.5 \% \\
37.5 \% \\
\end{array}$ \\
\hline $\begin{array}{l}\text { Initial presentation: } \\
\text {-Cough } \\
\text {-Expectoration } \\
\text {-Hemoptysis } \\
\text {-Dyspnea } \\
\text {-Chest pain } \\
\text {-Toxic findings } \\
\text {-Fever }\left(\geq 38^{\circ} \text { c) }\right. \\
\text {-Tachypnea (Breath/min. >20) } \\
\text {-Signs of Consolidation } \\
\end{array}$ & $\begin{array}{c}14 \\
14 \\
5 \\
6 \\
6 \\
11 \\
9 \\
13 \\
10 \\
\end{array}$ & $\begin{array}{c}93.3 \% \\
93.3 \% \\
33.3 \% \\
40 \% \\
40 \% \\
73.3 \% \\
60 \% \\
86.7 \% \\
66.7 \% \\
\end{array}$ \\
\hline
\end{tabular}

$\mathrm{M} \pm \mathrm{SD}=$ Mean \pm standard deviation

Table (2) Radiological findings of all studied patients

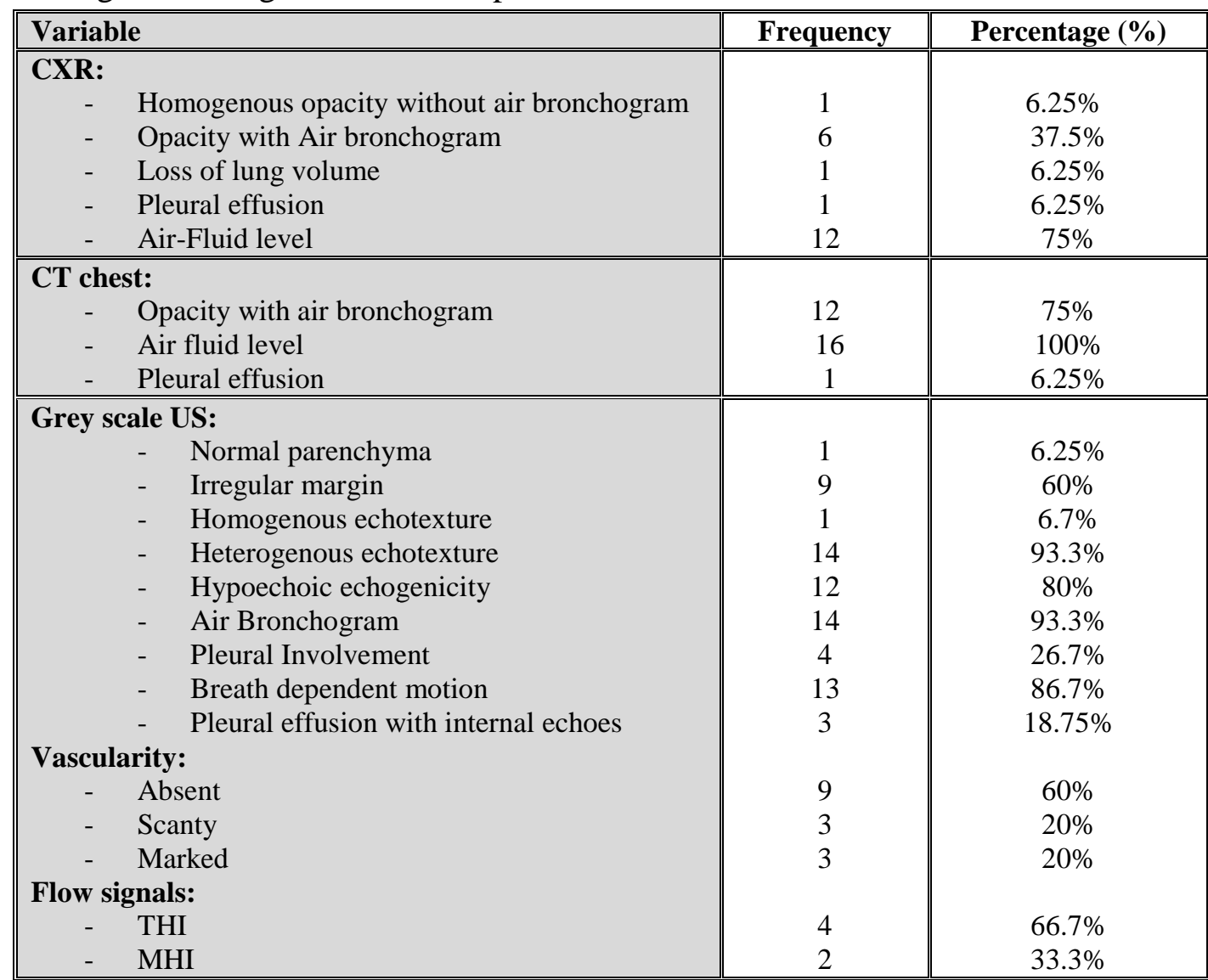

Sensitivity, specificity, PPV, NPV and DA of CXR, chest CT and grey scale US regarding diagnosis of lung abscess were compared (Table 3). Chest CT revealed the highest DA (98.5\%) with 100\% sensitivity, 98.3\% specificity, $88.9 \%$ PPV and $100 \%$ NPV. The sensitivity, specificity, PPV, NPV and diagnostic accuracy of chest X ray were 75\%, 98.3\%, 85.7\%, 96.6\% and 95.5\% respectively. Grey scale US had the least sensitivity (53.3\%), specificity was 98.2\%, PPV 96.7\%, NPV 67.7\% and DA 93.1\%. 
Table (3) Calculated sensitivity, specificity, PPV, NPV and accuracy of chest X ray, CT and grey scale US in diagnosis of abscess

\begin{tabular}{|l||l|l|l||l||l|}
\hline Lesion & Sensitivity & Specificity & PPV & NPV & Accuracy \\
\hline CXR & $75 \%$ & $98.3 \%$ & $85.2 \%$ & $96.6 \%$ & $95.5 \%$ \\
\hline \hline CT & $100 \%$ & $98.3 \%$ & $88.9 \%$ & $100 \%$ & $98.5 \%$ \\
\hline \hline US & $53.3 \%$ & $98.2 \%$ & $96.7 \%$ & $67.7 \%$ & $93.1 \%$ \\
\hline
\end{tabular}

CXR: Chest X ray, CT: computed tomography, US: Ultrasonography. PPV: Positive predictive value,

NPV: Negative predictive value. Analysis made by qui square test

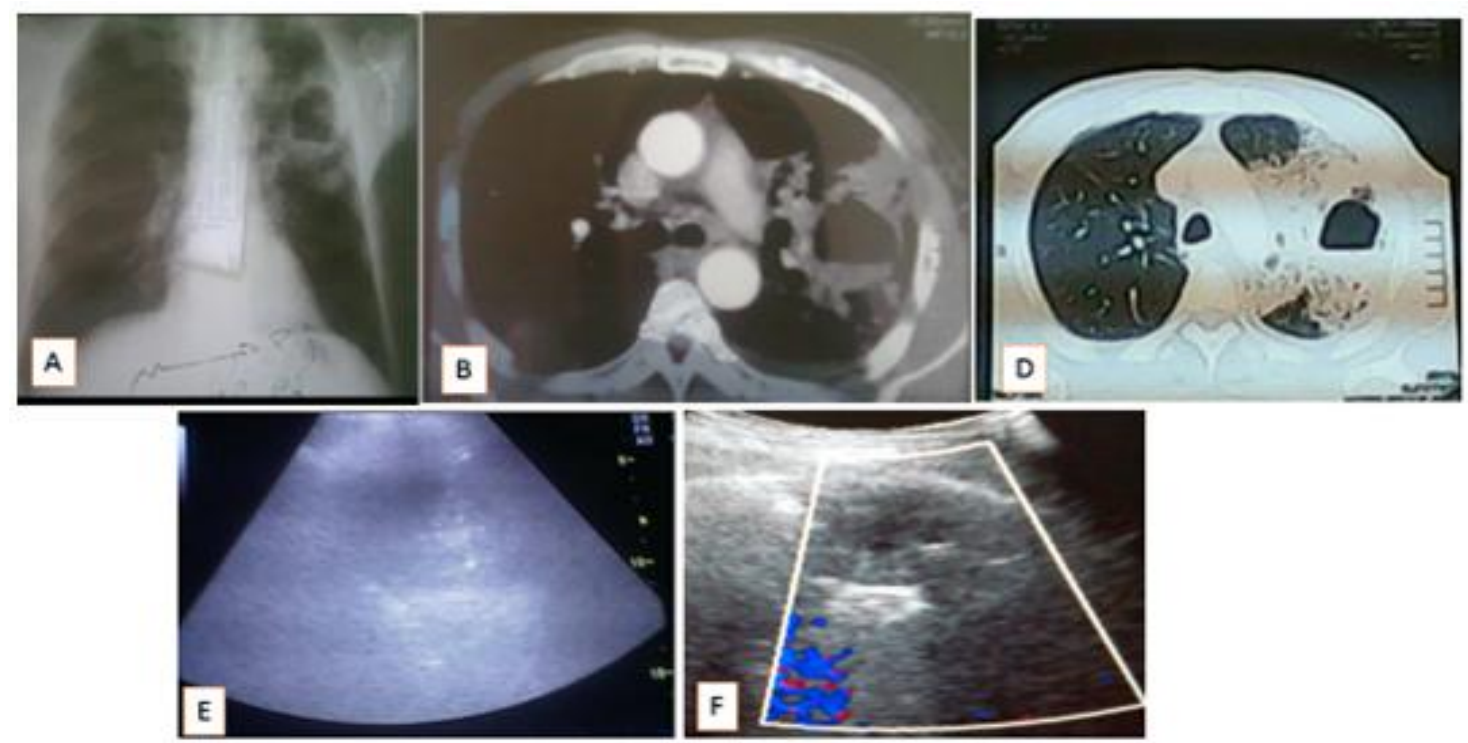

Fig.1: Lung consolidation with abscess formation. Male patient 4Sy old - A) CXR PA view revealed segmental consolidation with regular wall cavitary lesion with air fluid level at the lingula. B) Axial post contrast CT mediastinal window and D) pulmonary widow revealed thin wall cavity with air fluid level within a consolidation area of the left lung. E) US examination Grey scale and F) Color Doppler showed anechoic area with no color flow within

\section{Discussion}

Most of patients were presented by cough and expectoration (about 93\%). Associated tachypnea was present in about $87 \%$ of cases. Toxic manifestations were present in about $73 \%$ of patients. About $67 \%$ of cases showed signs of consolidation on local chest examination. Sixty percent of patients were presented with fever. Dyspnea and chest pain were equally presented each in $40 \%$ of cases. About $33 \%$ of patients were presented by hemoptysis.

These findings are supported by Moreira et al., 2006 who reported that lung abscess presented with cough and fever and that cough can be productive and associated with foul smelling purulent sputum or less frequently accompanied with blood tinged sputum in $33 \%$ of cases and that on chest examination of cases of lung abscess there will be features of consolidation such as localized dullness during percussion and bronchial breathing.
Lung abscess was radiologically presented mainly by air fluid level which was apparent in $75 \%$ of CXR of lung abscesses and it was detected in CT in $100 \%$ of cases associated with opacity with air bronchogram apparent in $75 \%$ of cases while CXR was able to show these air bronchogram in $37.5 \%$ of cases and one CXR showed opacity devoid of air bronchogram and another one was associated with loss of lung volume. Chest X ray and CT showed associated pleural effusion in only one case. In concordance with the present results, Lange and Walsh, 2007 informed that on plain CXR most abscesses originate within areas of pneumonic consolidation and are marked by the development of a discrete area of low density necrosis and cavitation and rupture of an abscess into a draining bronchus produces a cavity with an air-fluid level and that $\mathrm{CT}$ allows earlier detection of abscess formation within areas of pulmonary consolidation than does the standard chest 
radiograph and this can explain why the cavities were more apparent in CT films than CXR films.

Moreover, Albert et al., 2009 described radiologic appearance of lung abscess as a peripheral cavity in the dependent lung regions. Also, Reynolds et al., 2010 \& Reed et al., 2010 reported that the usual CT findings of a lung abscess include a spherical cavity with a thick wall that destroys the surrounding lung.

Regarding grey scale US, lung abscesses showed irregular margin in $60 \%$ of cases. This agrees with Tsai et al., 2008 who described lung abscess to have a blurred outer margin. Islam \& Tonn, 2009also reported that the boundary wall of lung abscess adjacent to the pleura can be assertive or irregular. Results of Chen et al., 2009 showed irregular abscess wall in $91 \%$ of cases. The results also agree with Koenig et al., 2011 who reported that typical features of a peripheral abscess demonstrate irregular hyperechoic borders abutting the surface of the pleura at an acute angle. Yang, 1997 said that lung abscess can be identified at US having a well-defined or irregular wall.

Heterogenous echotexture was found in more than $90 \%$ of abscess lesions and these results are supported by Yang et al., 1991 who reported that the dependent fluid portion of the abscess is in homogenous and Koenig et al., 2011 who reported that the abscess cavity will be heterogeneous with relatively anechoic areas with echogenic foci, corresponding to both air and tissue necrosis.

Eighty percent of abscess lesions showed hypoechoic echogenicity and this is also in concordance with Koh et al., 2002 who documented that lung abscess can be seen at US as a hypoechoic area and Bouhemad et al., 2007 who also reported that lung abscess appears as rounded hypo-echoic lesions with outer margins. Moreover, Sartori and Tombesi, 2010 who reported that lung abscesses typically appear as a largely anechoic lesions and

In the early stages of lung abscess, small abscesses are seen as pathological collections of fluid in the consolidated liver-like infiltrate
(Mathis, 2007) and this can explain the presence of air bronchogram in $93.3 \%$ of lung abscess lesions.

Twenty percent of cases of lung abscess were associated with pleural effusion with internal echoes. Sartori and Tombesi, 2010 reported that a small pleural effusion is commonly associated with lung abscesses.

Pleural involvement was seen in $26.7 \%$ of lung abscess lesions. Reissig and Kroegel, 2007 described pleural affection of pneumonia that the pleural line can appear interrupted, fragmented, and hypoechoic. Caiulo et al., 2013 found pleural line abnormalities in $20.2 \%$ of patients with pneumonia. Lung abscess usually develop on top of consolidating lung lesion and this also explain the possibility of pleural affection.

Majority of cases showed positive breath dependent motion. Lichtenstein and Menu, 1995 described normal lung anatomy with positive lung sliding sign.

Absent vascularity was shown in $60 \%$ of cases, while scanty and marked vascularity each was present in $20 \%$ of cases. Reissig et al., 2012 informed that necrotizing pneumonia is characterized by absent colour flow signals and this also can explain the absent vasculature in abscess lesion. But vasculature can be detected in the pericavitary consolidation as in the current study and this is in agree with Chen et al., 2009 who found that identification of color Doppler ultrasound vessel signals in pericavitary consolidation was the most useful and specific for identifying lung abscesses and differentiating it from empyema.

Regarding flow signals, $66.7 \%$ showed THI flow and 33.3\% showed MHI flow. Civardi et al., 1993 showed that benign lesions had a triphasic pattern derived from pulmonary arteries. The pulmonary artery has a predominantly high-impedance flow pattern (Spirathi and Mahajan, 2013). Also, Yuan et al., 1994 reported that benign lesions usually have high impedance flow.

Chest $\mathrm{X}$ ray was found to give a sensitivity and specificity of $75 \%$ and $98.3 \%$ respectively with 
DA of $95.5 \%$, PPV of $85.7 \%$ and NPV of $96.6 \%$. Stark et al., 1983 studied 70 inflammatory lesions in 63 patients (12 lung abscesses and 58 empyema) and diagnostic information not available from conventional CXR was obtained in $47 \%(33 / 70)$ of cases.

Sensitivity, specificity and diagnostic accuracy of chest CT were 100\%, $98.3 \%$ and $98.5 \%$ respectively with PPV of $88.9 \%$ and NPV of $100 \%$. Agree with these results are the results of the study performed by Stark et al., 1983 in which chest CT was able to diagnose $100 \%$ of lung abscess cases.

Chest ultrasound showed a sensitivity of $53.3 \%$, specificity of $98.2 \%$ with diagnostic accuracy of 93.1\%. PPV and NPV were $96.7 \%$ and $67.7 \%$ respectively. Better than these results, in Yang et al., 1991 study as $94 \%$ of abscess cases were demonstrated at US. This can be attributed to different size of lesions and different number as they studied 35 cases while abscess lesions were represented in this study in only 16 lesions.

\section{Conclusion}

The most frequent clinical presentations of lung abscess were cough \& expectoration, followed by tachypnea, toxic manifestations, then signs of consolidation, fever, dyspnea, chest pain, haemoptysis, and finally clubbing, signs of collapse and signs of pleural effusion.

The most common CXR findings were air-fluid level, followed by opacity with air bronchogram, then loss of lung volume and pleural effusion. Chest CT showed air-fluid level in all cases, then opacity with air bronchogram and associated pleural effusion. Grey scale US showed air bronchogram in most cases, then breath dependent motion, pleural involvement and pleural effusion with internal echoes, while one case $(6.75 \%)$ showed normal lung parenchyma.

Chest CT showed the highest diagnostic accuracy regarding diagnosis of lung abscess followed by CXR then grey scale ultrasound. Grey scale US has a relatively low sensitivity for diagnosis of lung abscess. It can be helpful in certain situation especially helpful if combined with color-Doppler sonographic examination.

\section{Recommendation}

On the basis of the current study, it is recommended to use chest $\mathrm{CT}$ for accurate diagnosis of lung abscess. It is also recommended to use grey scale US in patients who are susceptible to the adverse effects of radiation, such as pregnant women, children, and in patients with limited mobility, such as those in intensive care units.

\section{References}

1. Albert RK., Spiro SG., Jett JR. (2009): Infectious diseases. Bacterial pneumonia. In: Clinical respiratory medicine; Rabbat A., Huchon GJ. (eds), $3^{\text {rd }}$ ed., Mosby, Inc., an affiliate of Elsevier Inc., Philadelphia. Ch 7. PP: 333-350.

2. Biederer J., Wildberger J., Bolte H., et al., (2008): Protokollempfehlungen für die Computertomographie der Lunge: Konsensus der Arbeitsgemeinschaft Thoraxdiagnostik der DRG. Rofo; 180:471-479.

3. Bouhemad B., Zhang M., Lu Q., Rouby J. (2007): Clinical review: Bedside lung ultrasound in critical care practice. Crit Care; 11(1): 205.

4. Caiulo VA., Gargani L., Caiulo S., Fisicaro A., Moramarco F., Latini G. (2013): Lung ultrasound characteristics of community-acquired pneumonia in hospitalized children. Pediatr Pulmonol; 48:280-287.

5. Chen HJ., Yu YH., Tu CY., Chen CH., Hsia TC., Tsai KD., Shih CM., Hsu WH. (2009): Ultrasound in Peripheral Pulmonary Air-Fluid Lesions: Color Doppler Imaging as an Aid in Differentiating Empyema and Abscess. Chest; 135(6):1426-1432.

6. Civardi G., Fornari F., Cavanna L., Di Stasi M., Sbolli G. et al., (1993): 
Vascular signals from pleural-based lung lesions studied with pulsed Doppler ultrasonography. J Clin Ultrasound; 21:617-622.

7. Islam S. and Tonn H. (2009). Thoracic ultrasound overview. In: Bolliger CT, Herth FJF, Mayo PH, Miyazawa T, Beamis JF, eds. Clinical Chest Ultrasound. Sidney: Karger; p. 11-20.

8. Koenig SJ., Narasimhan M., Mayo PH. (2011): Thoracic Ultrasonography for the Pulmonary Specialist. Chest; 140(5):13321341.

9. Koh DM., Burke S., Davies N., Padley SPG. (2002): Transthoracic US of the Chest: Clinical Uses and Applications. RadioGraphics; 22, e1-.

10. Kuhajda I., Zarogoulidis K., Tsirgogianni K.,Tsavlis D., TsavlisD., Kosmidis C.,Tsakiridis K., Mpakas A.,Zarogoulidis P.,ZissimopoulosA., Baloukas D.,Kuhajda D. (2015): Lung abscess-etiology, diagnostic and treatment options. Ann Transl Med; 3(13):183.

11. Lange S., Walsh G. (2007): Infection and inflammatory disorders. In: Radiology of chest diseases, $3^{\text {rd }}$ ed., Grammlich, Plezhausen, Germany.ch 3. PP 64-109.

12. Lichtenstein DA. and Menu YA. (1995): Bedside Ultrasound Sign Ruling out Pneumothorax in the Critically III: Lung Sliding. Chest; 108:1345-1348.

13. Mathis G (2007): Subpleural Lung Consolidations

(Inflammatory consolidations in the lung). In: Chest sonography, $2^{\text {nd }}$ edition, Springer-Verlag Berlin Heidelberg. Ch 4. PP.50-63.

14. Moreira Jda S., Camargo Jde J., Felicetti JC., Goldenfun PR., Moreira AL., Porto Nda S. (2006): "Lung abscess: analysis of 252 consecutive cases diagnosed between 1968 and 2004". Jornal brasileiro de pneumologia: publicaçäo oficial da Sociedade Brasileira de Pneumologia e Tisilogia. 32 (2): 136-43.
15. Patradoon-Ho P. and Fitzgerald DA. (2007): Lung abscess in children. Paediatr Respir. Rev.; 8:77-84.

16. Reed A., Shogan P., Folio L. (2010): Radiology corner: Thoracic empyema versus lung abscess. Mil Med; 175(9): 701-702.

17. Reissig A, Gramegna A and Aliberti $S$ (2012): Review article. The role of lung ultrasound in the diagnosis and follow- up of community-acquired pneumonia. European Journal of Internal Medicine; 23(5):391-397.

18. Reissig A., Kroegel C. (2007): Sonographic diagnosis and follow-up of pneumonia: a prospective study. Respiration; 74:537-547.

19. Reynolds JH., McDonald G., Alton H., Gordon SB. (2010): Pneumonia in the immunocompetent patient. Br J Radiol; 83(996):998-1009.

20. Sartori S. and Tombesi P. (2010): Emerging roles for transthoracic ultrasonography in pulmonary diseases. World J Radiol; 2(6): 203-214.

21. Seo H., Cha SI., Shin KM., et al., (2013): Focal necrotizing pneumonia is a distinct entity from lung abscess. Respirology ; 18:1095-100.

22. Sripathi S. and Mahajan A. (2013): Comparative Study Evaluating the Role of Color Doppler Sonography and Computed Tomography in Predicting Chest Wall Invasion by Lung Tumors. Journal of ultrasound in medicine; 32 (9): 1539-1546.

23. Stark DD., Federle MP., Goodman PC., Podrasky AE., Webb WR. (1983): Differentiating Lung Abscess and Empyema: Radiography and Computed Tomography. AJR; 141:163-167.

24. Tsai TH, Jerng JS and Yang PC (2008): Clinical Applications of Transthoracic Ultrasound in Chest Medicine. J Med Ultrasound;16(1):7-25.

25. Wielpütz MO., Heußel CP., Herth FJF., 
Kauczor HU. (2014): Radiological diagnosis in lung disease. Factoring treatment options into the choice of diagnostic modality. Disch Arztebl Int; 111(11):181-187.

26. Yang PC (1997): Ultrasound-guided transthoracic biopsy of peripheral lung, pleural, and chest wall lesions. J Thorac Imaging; 12:272-284.

27. Yang PC., Luh KT., Lee YC., Chang DB., Yu CJ., Wu HD., Lee LN., Kuo SH. (1991): Lung abscesses: US examination and US-guided transthoracic aspiration. Radiology; 180(1):171-5.

28. Yuan A., Chang DB., Yu CJ., Kuo SM., Luh KT., Yang PC. (1994): Color Doppler sonography of benign and malignant pulmonoray masses. Am J Roentgenol; 163:545-549. 